\title{
Power minimization of cooperative beamforming networks with spectrum sharing
}

\author{
Cen Ling ${ }^{1}$, Xuefeng Yin ${ }^{1 *}$, Silvia Ruiz Boqué ${ }^{2}$ and Mario García-Lozano ${ }^{2}$
}

\begin{abstract}
Cooperative communication with spectrum sharing has been proved to be an efficient way to reduce energy consumption. In this work, an optimal power allocation algorithm is proposed for mobile cooperative beamforming networks, where several secondary users (SUs) collaboratively act as relay nodes to assist the transmission of a primary user (PU) in reward of transmitting their own information. The optimal power allocation scheme is obtained by solving a convex optimization problem aiming at minimizing network energy consumption with guaranteed quality of service (QoS) for both PU and SUs. Simulation results show that with the consideration of overheads, circuit power, and energy costs for message sharing, a cooperative beamforming network augmented with our proposed power allocation algorithm performs better in scenarios of more SUs in terms of higher energy efficiency and less power consumption per unit throughput. Additionally, the proposed scheme outperforms the conventional best-relay selection approach in the aspects of power consumption per unit throughput, energy efficiency, and network power consumption. Furthermore, simulations also demonstrate that the derived cooperative beamforming algorithm exhibits superior performance in energy saving than conventional methods in high-speed scenarios.
\end{abstract}

Keywords: Cooperative beamforming; Power minimization; Spectrum sharing; Mobile network

\section{Introduction}

Towards the fifth generation (5G) of wireless/mobile broadband communication, many advanced key technologies are going to be implemented, including spectrum sharing management, exploiting the Cloud-RAN (CRAN) and mobile clouds concepts as well as heterogeneous network coordination, in order to meet the demand for larger bandwidth, higher data rate up to Gbit/s and seamless connection access [1-3]. Furthermore, reduction of unnecessary energy consumption becomes one of the major concerns in 5G communications, which not only reduces environmental impact but also cuts overall network costs and helps make communication more practical and affordable. These studies belong to the research of 'green networking' which aims in increasing energy savings while maintaining satisfactory users' quality of service (QoS) [4-6]. Besides, cooperative techniques are promising in improving the reliability of the communications against channel impairments

\footnotetext{
*Correspondence: yinxuefeng@tongji.edu.cn

${ }^{1}$ College of Electronics and Information Engineering, Tongji University, 4800 Caoan Road, Shanghai, 201804, China
}

Full list of author information is available at the end of the article and achieved throughput through aggregating resources offered by different collaborative entities and realizing significant energy savings, targeting a so-called green and soft $5 \mathrm{G}$ system [7].

Apart from 'cell breathing' of base station coordination and clustered cooperative schemes [8,9], user cooperation, as a new form of spatial diversity, enables singleantenna mobiles in a multiuser environment to share their antennas and generate a virtual multiple-antenna transmitter, providing higher throughput, robustness to channel variations and ubiquitous mobile access for media-rich mobile devices. Recently, being extended with the concept of cognitive radio, user cooperation with spectrum sharing capability, i.e., secondary users (SUs) share the same spectrum with the primary user (PU), can further improve spectral efficiency. User-cooperative diversity protocols that combat fading induced by multipath propagation in wireless networks and the outage capacity characterization of various relaying protocols, i.e., fixed relaying, selective relaying, and incremental relaying, are characterized [10]. In literature, numerous criteria have been proposed for relay node selection based on channel conditions [11], the distances between nodes [12],

\section{黑 Springer}

(c) 2015 Ling et al: licensee Springer. This is an Open Access article distributed under the terms of the Creative Commons

Attribution License (http://creativecommons.org/licenses/by/4.0), which permits unrestricted use, distribution, and reproduction in any medium, provided the original work is properly credited. 
and the transmission contention [13], etc. It has been demonstrated in $[14,15]$ that when more relay nodes, also called as cooperators, are involved in transmission, less energy costs and higher network throughput can be achieved simultaneously, compared with the single-relay transmission. This motivated the research on methodologies of selecting multiple entities to act as cooperators, also called as 'cooperative beamforming. However, several issues should be considered from a media access control (MAC) layer perspective when multiple cooperating entities are involved. The first issue is that more control signaling overhead should be considered for selecting and coordinating multiple cooperating entities [16]. The second issue is related to users' willingness to cooperate. Furthermore, incentive mechanisms are of necessity for promoting user cooperation in order to prevent unwillingness and selfishness of the SUs via reputation evaluation, cost reduction, or increase of transmission time [17-20].

As the energy expenditure becomes a major concern for the operators of communication networks, constructing cooperative networks with the energy consumption minimized has become an important research field [21]. Multiple solutions of resource allocation were derived based on convex optimization or game theory via lowering power consumption under certain constraints, such as minimizing the outage probability [22-24], maximizing energy efficiency [25-28], maximizing overall throughput [29,30], maintaining service quality, or maximizing the sum of SUs' capacities and signal-to-noise power ratio (SNR) [31]. Joint relay selection and power allocation schemes are proposed by factoring the cost of acquiring channel state information (CSI) to minimize the total energy consumption [32]. The allocation schemes obtained are applicable to adapting the transmission power, bandwidth, time, and accessibility for the nodes involved in the cooperative network. In addition, circuit power consumption model has been taken into account in calculating the energy efficiency of cooperative heterogeneous and beamforming networks in $[25,33]$.

The aforementioned resource allocation methods share some common drawbacks which limit their applicabilities in cooperative spectrum sharing networks in cases where a PU and multiple SUs coexist. First, the energy consumption minimization strategies adopted in these methods usually consider a single relay node, which is typical merely for the best relay selection scheme. The adaptation of the strategies in the situation with multiple SUs selected simultaneously as relay nodes, as in the cooperative beamforming scenario, has not been investigated thoroughly so far. Furthermore, SUs may be reluctant to relay the traffic for the PU, if SUs do not forsee an immediate benefit or reward from their cooperation or they have some security and privacy concerns or encounter resource limitation, e.g., battery shortage [14]. This issue concerning the incentive mechanisms so as to prevent unwillingness and selfishness of the SUs has not been paid great attention in recent researches on cooperative beamforming networks. Moreover, the existing power allocation schemes are derived under an ideal channel assumption that channel coefficients for multiple links among the communication nodes are independent random variables following identical distributions. This leads to nonrealistic conclusions for performance evaluation. It is necessary to re-design the resource allocation methods by taking into account some new constraints posed by the existence of multiple relays, the energy costs of the communication coordination overheads, the influence of the rewards for the relay nodes, and realistic propagation scenarios.

In this paper, we propose an energy-saving strategy for cooperative beamforming by finding the optimal power allocation scheme. This method is particularly useful in cooperative beamforming for mobile spectrum sharing scenarios where a PU and multiple SUs co-exist. The proposed power allocation scheme is obtained by solving a convex optimization problem with the boundary conditions specifying the minimum QoS for the PU and SUs, similar to the approach described in [19]. A model of constant circuit power consumption originally introduced in [25] is considered for calculating the network power consumption. Simulations under the setting of realistic fast fading channels generated by using an infinite impulse response (IIR) filter demonstrate that the energy efficiency which is defined as the ratio of outage capacity over total energy consumed, i.e., a necessary extension from single-relay scenarios $[22,34]$ to multi-relay cooperative beamforming cases, can be significantly improved by implementing the proposed optimization scheme. Furthermore, the proposed scheme exhibits superior performance compared with some conventional methods including the best relay selection approach.

The rest of the paper proceeds as follows. In Section 2, the considered cooperative beamforming scenario in a cognitive network is introduced. In Section 3, the optimization method of power allocation and the derivation of energy efficiency for cooperative beamforming are presented. Simulation results are elaborated in Section 4 for performance assessment of the proposed scheme. Eventually, conclusive remarks are given in Section 5.

\section{System model}

Let us consider a wireless network where totally $M$ SUs act simultaneously as relay nodes for one PU, as illustrated in Figure 1. A direct propagation path exists between the primary transmitter (PT) and primary receiver (PR). Each node is equipped with a single antenna for signal transmission and reception. A dual-hop decode-andforward (DF) cooperative communication mode with 


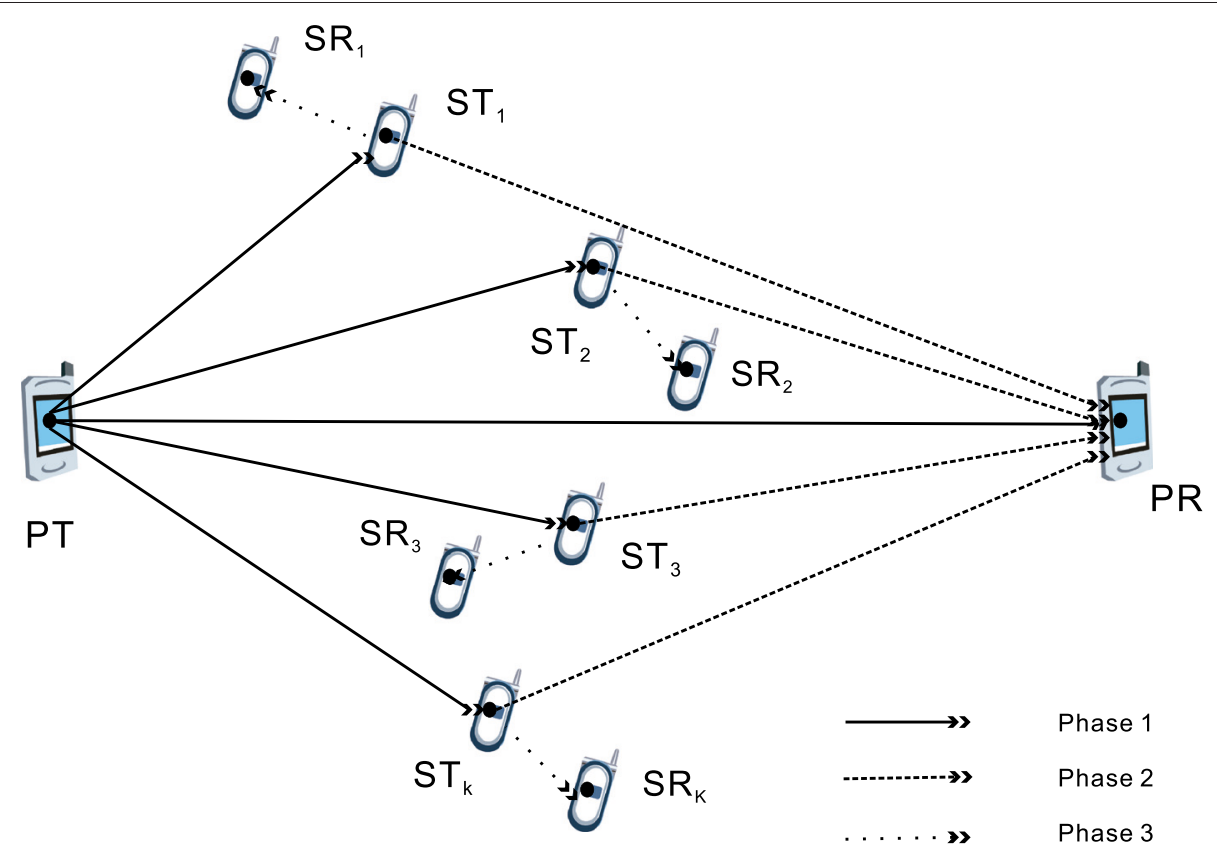

Figure 1 Cooperative beamforming model with three phases.

synchronization and diversity combining at the PR is considered. In return, the SU selected as a relay is rewarded with getting access to the channel for its own communication with a secondary receiver (SR) under the PU's authority.

Resource allocation can be performed with a frame or a slot structure according to the system design in a code division multiple access (CDMA) system, similar to Bluetooth, Wi-Fi, or sensor networks. The cooperative communication with all SUs and PU involved can be performed in three phases, as illustrated in Figure 2, where $T$ and $t_{k}$ denote the transmission time assigned to the PU and the SUs, respectively [35]. In the first phase, the PT broadcasts the data to the PR and all $M$ SUs that could act as relays. In the second phase, the SUs decode the received signals individually and forward to the PR, acting as secondary transmitters (STs). At the PR, the signals from the PT and all STs are soft-combined and decoded. An ST's own transmission to its SR is performed during a short period $t_{k}$ in the third phase. The overall time for completing these phases is then calculated as $T+t_{k}=\beta_{k} T$, where $\beta_{k} \geq 1$ can be determined optimally as a balance between

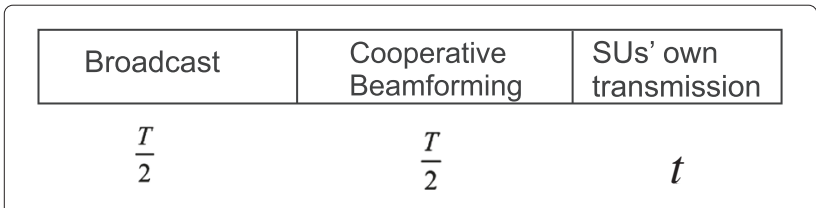

Figure 2 Time slot allocation for primary and secondary user. the ST's throughput and power consumption, as will be discussed later in this paper.

Noted that before the foregoing three phases, a short time period is usually allocated for the transmission of training signals and the estimation of CSI. The signal received for a link between any pair of nodes $i$ and $j$, can be written as:

$$
y_{j}=h_{i j} \sqrt{P_{i j}} x_{i}+n_{j},
$$

where $h_{i j}$ is the complex channel coefficient from the node $i$ to the node $j, P_{i}$ denotes the transmitting power of the node $i$ to the node $j$ over the transmission bandwidth $W$, and $n_{j}$ is the thermal noise generated by the node $j$ and is modeled as an additive white Gaussian random variable with zero mean and spectral density height of $N_{o}(\mathrm{~W} / \mathrm{Hz})$. The complex channel coefficient $h_{i j}$ is assumed to be a complex circularly symmetric Gaussian random variable with magnitude $\left|h_{i j}\right|$ following a Rayleigh distribution. The mean of the Rayleigh distribution is dependent on the distance $d_{i j}$ between the $i$ th node and the $j$ th node according to $d_{i j}^{-\alpha}$, where $\alpha$ is an exponent constant describing the attenuation caused by the wave propagation. It is easy to show that $\left|h_{i j}\right|^{2}$ follows an exponential distribution with its rate parameter being $\lambda_{i j}=d_{i j}^{\alpha}$ [22]. From Equation 1, the SNR for the signals received at the node $j$ when the node $i$ transmits can be calculated as:

$$
\mathrm{SNR}_{i j}=\frac{P_{i j}\left|h_{i j}\right|^{2}}{N_{0}}, \quad \gamma_{i j} \triangleq \frac{P_{i j}}{N_{0}},
$$


where $\gamma_{i j}$ is defined as the ratio between the transmission signal power over the noise variance.

In order to successfully decode the information received by $\mathrm{ST}_{k}$, the $\mathrm{SNR}$ of the link between $\mathrm{PT}$ and $\mathrm{ST}_{k}$, i.e., $\mathrm{SNR}_{p s_{k}}$ is usually required to be higher than a threshold $\theta_{1}$, i.e.,

$$
\mathrm{SNR}_{p s_{k}}=\frac{P_{p s_{k}}\left|h_{p s_{k}}\right|^{2}}{N_{0}}=\frac{P_{p s_{k}} d_{p s_{k}}^{-\alpha}}{N_{0}}>\theta_{1},
$$

where $d_{p s_{k}}$ represents the distance from the $\mathrm{PT}$ to $\mathrm{ST}_{k}$, $P_{p s_{k}}$ denotes the transmission power of the PT, and $h_{p s_{k}}$ is the channel coefficient of the link between the PT and $\mathrm{ST}_{k}$. Applying the triangular principle for $d_{p s_{k}}$, the distance $d_{p}$ from the PT to the PR, and the distance $d_{s_{k}} d$ from $\mathrm{ST}_{k}$ to the PR, it is easy to show that $d_{p s_{k}}$ should be confined within the range described by the following inequalities:

$$
\left|d_{p}-d_{s_{k} d}\right|<d_{p s_{k}}<\min \left\{\left(d_{p}+d_{s_{k} d}\right),\left(\frac{P_{\max }}{N_{0} \theta_{1}}\right)^{\frac{1}{\alpha}}\right\}
$$

where $P_{\max }$ represents the maximum transmission power of the network. Notice that Equation 4 can also be applied as a fundamental principle for relay selection when multiple STs are available in the network. In order to improve the understandings, explanations of the adopted symbols are demonstrated in Table 1.

In the following, an analytical expression is derived for the transmission rate of the cooperative communication system illustrated in Figure 1. In the first phase shown in Figure 2, the PT directly transmits to the PR without spectrum cooperation. The data rate per $\mathrm{Hz} R_{d}$ for the link between the PT and the PR can be calculated as:

$$
R_{d}=\log _{2}\left(1+\gamma_{d}\left|h_{d}\right|^{2}\right),
$$

where $\gamma_{d}$ denotes the ratio of the direct transmitting power $P_{d}$ of the PT to the noise variance and $h_{d}$ is the channel coefficient for the direct link from the PT to the PR.

In the second phase, cooperative beamforming is performed together with the direct transmission from the PT to the PR. The STs forward the signals to the PR after the decoding step, and the PR combines the signals originally transmitted by the PT and all STs using, e.g., the maximal ratio combining (MRC). The maximum data rate per second at the PR can be calculated by considering that all $M$ STs transmit during the time $T / 2$ to be:

$$
R_{\mathrm{c}}=\min _{k \in \kappa}\left\{R_{p s_{k}}, R_{\mathrm{MRC}}\right\},
$$

with $k$ being the indices of the STs, $\kappa \in\{1,2, \ldots, M\}$, $R_{p s_{k}}$ representing the data rate between the $\mathrm{PT}$ and $\mathrm{ST}_{k}$, and $R_{\mathrm{MRC}}$ denoting the combination rate at the PR of the signals arriving from the PT and all STs. They can be calculated as respectively:
Table 1 Explanation of adopted symbols

\begin{tabular}{ll}
\hline Symbol & Explanation \\
\hline$y_{j}$ & Received signal at the $j$ th node \\
$h_{i j}$ & Channel coefficient from ith to $j$ th node \\
$P_{i j}$ & $\begin{array}{l}\text { Transmitting power per bandwidth from the } i \text { th node to the } \\
\text { jth node }\end{array}$ \\
$x_{i}$ & Transmitting signal at the ith node \\
$W_{N}$ & Transmission bandwidth \\
$N_{0}$ & Spectral density height of thermal noise \\
$n_{j}$ & Thermal noise \\
$\lambda_{i j}$ & Rate parameter of exponential distribution \\
$d_{i j}$ & Distance between the ith and jth nodes \\
$R_{d}$ & Direct transmission data rate \\
$\gamma_{d}$ & Transmitting SINR of direct transmission \\
$h_{d}$ & Channel coefficient of direct transmission \\
$R_{C}$ & Data rate of PR \\
$R_{p s_{k}}$ & Data rate of the link of PT-SR \\
$R_{\mathrm{MRC}}$ & Data rate of MRC
\end{tabular}

$\gamma_{p} \quad$ Ratio between the transmission signal power over the noise variance for PU

Channel coefficient of the link PT-SR $k$

Ratio between the transmission signal power over the noise variance between $\mathrm{ST}_{k}$ and $\mathrm{PR}$

Channel coefficient of the link ST $T_{k}-P R$

Data rate of the kth SU

Ratio between the transmission signal power over the noise variance for the $k$ th SU

Channel coefficient of the link ST-SR

Cooperative network throughput

Time for the 1st and 2nd phase transmission

Time for the third phase transmission

Transmitting power of PT

Transmitting power of non-cooperative scheme

Transmitting power of the $\mathrm{ST}_{k}$

Transmitting power of the third phase

Beamforming weight of the $\mathrm{ST}_{k}$

Circuit power

Overheads of cooperative beamforming

Overheads of message sharing

Circuit power

Number of cooperative SUs

Number of bits per symbol for cooperative beamforming

Number of bits per symbol for message sharing

Amplifier efficiency

Number of symbols of training period

Number of symbols of cooperative beamforming

Power consumption per throughput 
Table 1 Explanation of adopted symbols (Continued)

\begin{tabular}{ll}
\hline Symbol & Explanation \\
$\beta_{\mathrm{k}}$ & Time allocation ratio of the $k$ th SU \\
$Q_{p}$ & QoS of PU \\
$Q_{s}$ & QoS of SU \\
$P_{d}^{\text {out }}$ & Outage probability of direct transmission \\
$C_{d}^{\text {out }}$ & Outage capacity of direct transmission \\
$C_{\text {Sut }}^{\text {out }}$ & Outage capacity of SU \\
$\varepsilon$ & Outage probability threshold \\
$C_{\mathrm{cb}}^{\text {out }}$ & Outage capacity of cooperative beamforming \\
$\eta$ & Energy efficiency \\
$\theta_{1}$ & SNR threshold for decoding \\
$\theta_{2}$ & SNR of the worst link between ST $\mathrm{k}$ and PR \\
$d_{p}$ & Distance between PT and PR \\
$d_{s k}$ & Distance between PT and PR \\
$d_{s u}$ & Distance between ST and SR \\
$d_{p s_{k}}$ & Distance between PT and ST \\
$P_{\text {max }}$ & Maximum transmitting power \\
$\gamma_{d}$ & Ratio between the direct transmission signal power over the \\
$\zeta$ & noise variance \\
$L_{s}$ & Network energy consumption for unit bit of throughput \\
$v$ & Number of SUs \\
\hline & Moving speed of users \\
\hline
\end{tabular}

$$
\begin{aligned}
R_{p s_{k}} & =\frac{1}{2} \log _{2}\left(1+\gamma_{p}\left|h_{p s_{k}}\right|^{2}\right) \\
R_{\mathrm{MRC}} & =\frac{1}{2} \log _{2}\left(1+\gamma_{p}\left|h_{d}\right|^{2}+\sum_{k=1}^{M} \gamma_{s_{k} d}\left|h_{s_{k} d}\right|^{2}\right)
\end{aligned}
$$

where $\gamma_{p}$ denotes the ratio of the transmission power $P_{p}$ of the PT to the noise variance $N_{0}, \gamma_{s_{k}}$ represents the ratio of the transmission power of the $\mathrm{ST}_{k}$ and $N_{0}$, and $h_{s_{k} d}$ is the channel coefficient for the link between the $\mathrm{ST}_{k}$ and the PR.

In the last phase after the cooperative beamforming is executed, each ST gets the permission to use the channel between $\mathrm{ST}_{k}$ and $\mathrm{SR}_{k}$. The data transmission rate can be calculated as:

$$
R_{s u_{k}}=\log _{2}\left(1+\gamma_{s u_{k}}\left|h_{s u_{k}}\right|^{2}\right)
$$

where $\gamma_{s u_{k}}$ and $h_{s u_{k}}$ denote respectively the ratio of transmission power of the $\mathrm{ST}_{k}$ and the noise variance and the channel coefficient of the link between the $\mathrm{ST}_{k}$ and the $\mathrm{SR}_{k}$.

The total network's throughput $R_{\mathrm{N}}$ can then be calculated as:

$$
R_{N}=\frac{T R_{c}+\sum_{k=1}^{M} t_{k} R_{s u_{k}}}{\bar{t}+T}
$$

where $t_{k}$ is the time utilized in the third phase for the $k$ th $\mathrm{ST}$, and $\bar{t}$ represents the average of $t_{k}, k=1, \ldots, M$. In the system considered here, every SU transmits its own information in its allocated channel, and the individual transmission time $t_{k}$ is determined using an optimization algorithm described in the later sections of the paper.

The network's power consumption $P_{N}$, as a holistic and system-wide metric, which includes all the energy consumption such as transmission power, circuit power, and signaling overhead in the entire network, can be calculated as:

$P_{N}=\frac{\frac{T}{2}\left(P_{p}+\sum_{k=1}^{M} w_{k} P_{s_{k}}\right)+\sum_{k=1}^{M} w_{k} t_{k} P_{s u_{k}}}{\bar{t}+T}+P_{\mathrm{OV}}$,

where the optimal beamforming weight $w_{k}$ for the $k$ th ST can be calculated as $\left|h_{s_{k} d}\right| /\|\boldsymbol{h}\|$ with $\|\cdot\|$ being the norm of given vector and $\boldsymbol{h}=\left[\left|h_{s_{1} d}\right|,\left|h_{s_{2} d}\right|, \ldots,\left|h_{s_{M} d}\right|\right]^{T}$ representing the vector consisting of the channel gains from the STs to the PR and $P_{\mathrm{OV}}$ denotes the power overheads required in the source message sharing and channel estimation phases [25].

The overhead consumption $P_{\mathrm{OV}}$ is obtained by using the constant circuit-power consumption model [25]:

$P_{\mathrm{OV}}=\left(\frac{P_{\mathrm{SR}}}{\rho}+(1+M) P_{c}\right) \frac{b_{\mathrm{RD}}}{b_{\mathrm{SR}}}+\left(\frac{P_{\mathrm{TR}}}{\rho}+(1+M) P_{c}\right) \frac{N_{\mathrm{TR}}}{N_{\mathrm{RD}}}$,

where $P_{\mathrm{TR}}$ denotes the power used for the training signals, $P_{\mathrm{SR}}$ signifies the overhead power of message sharing period, $\rho$ represents the amplifier efficiency, $P_{c}$ is the circuit power, $b_{\mathrm{RD}}$ and $b_{\mathrm{SR}}$ denote respectively the number of bits per symbol during the cooperative beamforming period and during the message sharing period, $W$ is the available transmission bandwidth, and finally, $N_{\mathrm{TR}}$ and $N_{\mathrm{RD}}$ represent the number of symbols transmitted during the two periods, respectively. $P_{\mathrm{TR}}$, is selected such that the SNR of the worst link between the $\mathrm{ST}_{k}$ and $\mathrm{PR}$ is $\theta_{2}$. In addition, $P_{\mathrm{SR}}$ can be calculated as:

$$
\begin{gathered}
P_{\mathrm{SR}}=\frac{\gamma_{\text {th }}\left(b_{\mathrm{SR}}\right) N_{0} W}{\min \left(\left|h_{p s_{k}}\right|^{2}\right)}, \\
\gamma_{\text {th }}\left(b_{\mathrm{SR}}\right)=-\frac{1}{c_{2}}\left(2^{b_{\mathrm{SR}}}-1\right) \ln \left(\frac{p_{\mathrm{th}}}{c_{1}}\right), \\
p_{\text {th }}(\mathrm{b})=c_{1} \exp \left(-\frac{c_{2} p_{p}}{\left(2^{b}-1\right) N_{o} W}\right),
\end{gathered}
$$

where $\gamma_{\text {th }}\left(b_{\mathrm{SR}}\right)$ is the SNR required to achieve the target $\mathrm{M}$-ary quadrature amplitude modulation (M-QAM) bit error probability, $p_{\text {th }}$ and $c_{1}=0.2, c_{2}=1.5$.

To compute the average transmission rates for the entire time period ( $T+t_{k}=\beta_{k} T$ ), we consider that the cooperative communication is performed within $1 / \beta_{k}$ of the 
whole time period, and the secondary communication utilizes the spectrum in the rest of time, i.e., $\left(\beta_{k}-1\right) / \beta_{k}$ of the whole period. The average transmission rates of $\mathrm{PU}$ in the first and second phases and of SUs in the third phase can then be calculated as, respectively:

$$
\bar{R}_{p}=\frac{R_{\mathrm{c}}}{\bar{\beta}_{k}}, \bar{R}_{s u}=\sum_{k=1}^{M} \frac{\beta_{k}-1}{\beta_{k}} R_{s u_{\mathrm{k}}} \text { with } k=1, \ldots, M .
$$

\section{An algorithm for energy consumption minimization and cooperative beamforming \\ 3.1 Optimal power allocation for cooperative beamforming}

We now derive an algorithm for determining the optimal power allocation with minimal energy consumption for the considered cooperative beamforming system. It is reasonable to assume that within the channel coherence time, CSI, correctly detected by corresponding UEs, has been sent to the PT. Based on the CSI, the transmission powers, i.e., $P_{p}, P_{s_{k} d}$, and $P_{s u_{k}}$, shall be determined with the objective to minimize the average energy consumption of the network while guaranteeing users' QoS. To be specific, $P_{s_{k} d}$ denotes the transmission power of the $k$ th ST to the PR, and $P_{s u_{k}}$ represents the transmission power of the $\mathrm{SU}_{k}$ 's own transmission in the third phase. The predefined QoS of the PU and SU denoted with $Q_{p}$ and $Q_{s}$, respectively, are identified with the required transmission rate. The optimization problem can be formulated as:

$$
\begin{aligned}
& \min _{P, \beta_{k}} T \cdot\left(\frac{1}{2 \beta_{k}}\left(P_{p}+P_{s_{k} d}\right)+\frac{\beta_{k}-1}{\beta_{k}} P_{s u_{k}}\right), \\
& \text { s.t. } \frac{1}{2 \beta_{k}}\left(\log _{2}\left(1+\frac{P_{p}\left|h_{p}\right|^{2}}{N_{o}}\right)+\log _{2}\left(1+\frac{P_{s_{k} d}\left|h_{s_{k} d}\right|^{2}}{N_{o}}\right)\right) \geq Q_{p},
\end{aligned}
$$

$\frac{\beta_{k}-1}{\beta_{k}} \log _{2}\left(1+\frac{P_{s u_{k}}\left|h_{s u_{k}}\right|^{2}}{N_{o}}\right) \geq Q_{s}$,

$\beta_{k}>1$.

The inequalities Equations 16 and 17 represent respectively the QoS requirements of the PU and of the SU. The left-hand sides of Equations 16 and 17 separately denote the transmission rate for PU during the cooperative communication (phases 1 and 2) and SU's own transmission (phase 3).

This convex optimization problem in Equations 15 to 18 satisfies the Karush-Kuhn-Tucker conditions, and it can be solved using the Lagrangian equation [36,37]. Besides, $T$ is normalized as 1 . Detailed convexity analysis and the solution of the optimization problem are given in Appendix 1 and Appendix 2, respectively.
Despite the unawareness of $\beta_{k}$, the optimal powers can be computed as, respectively:

$$
\begin{aligned}
& P_{p}=N_{o}\left(\frac{2^{\beta_{k} Q_{p}}}{\left|h_{p}\right|\left|h_{s_{k} d}\right|}-\frac{1}{\left|h_{p}\right|^{2}}\right), \\
& P_{s_{k} d}=N_{o}\left(\frac{2^{\beta_{k} Q_{P}}}{\left|h_{p}\right|\left|h_{s_{k} d}\right|}-\frac{1}{\left|h_{s_{k} d}\right|^{2}}\right), \\
& P_{s u_{k}}=\frac{N_{o}}{\mid h_{\left.s u_{k}\right|^{2}}}\left(2^{\frac{\beta_{k} Q_{s}}{\beta_{k}-1}}-1\right) .
\end{aligned}
$$

Once these optimal transmission powers are known, the optimal $\beta_{k}$ can be calculated by solving the function:

$$
\begin{gathered}
\frac{2^{\beta_{k} Q_{p}}}{\left|h_{s_{k} d}\right|\left|h_{p}\right|}\left(Q_{p} \beta_{k}-1\right)-\frac{\beta_{k} Q_{s} 2^{\frac{\beta_{k} Q_{s}}{\beta_{k}-1}}}{\left|h_{s u_{k}}\right|^{2}\left(\beta_{k}-1\right)} \\
+\frac{2^{\frac{\beta_{k} Q_{s}}{\beta_{k}-1}}-1}{\left|h_{s u_{k}}\right|^{2}}+\frac{1}{2\left|h_{p}\right|^{2}}+\frac{1}{2\left|h_{s_{k} d}\right|^{2}}=0 .
\end{gathered}
$$

It can be readily shown in Appendix 2 that the lefthand side of Equation 20 has the derivative with respect to $\beta_{k}$ always larger than zero and, hence, increases monotonically along with $\beta_{k}$. Thus, the optimal $\beta_{k}$ can be determined by applying the Newton's iterative method as described in [38].

Notice that when the cooperative beamforming is not executed, the optimization problem for the power allocation via minimizing the energy consumption reduces to;

$$
\min \left\{P_{d}\right\} \quad \text { s.t. } \log _{2}\left(1+\frac{P_{d}\left|h_{d}\right|^{2}}{N_{o}}\right) \geq Q_{p} .
$$

The optimal power for the direct transmission in this case can be calculated as:

$$
P_{d}=\frac{1}{\left|h_{d}\right|^{2}}\left(e^{Q_{p}}-1\right) \text {. }
$$

This scheme is used as a reference for evaluating the performance of the proposed method in simulations.

\subsection{Energy efficiency of the cooperative beamforming scheme}

The expressions of the outage probability and outage capacity for the proposed cooperative beamforming scheme are derived in the following. It is easy to show that during the direct transmission from the PT to the PR, the outage probability between the PT and PR can be calculated as:

$$
P_{d}^{\text {out }}=1-\exp \left(-d_{d}^{\alpha} \cdot \frac{2^{R}-1}{\gamma_{d}}\right) .
$$


Hence, for a fixed outage probability $\varepsilon$, the outage capacity can be obtained as:

$$
C_{d}^{\text {out }}=\log _{2}\left(1+\frac{\gamma_{d}}{d_{d}^{\alpha}} \ln \frac{1}{(1-\varepsilon)}\right)
$$

In the third phase, the outage capacity for an SU can be formulated similarly as:

$$
C_{s u}^{\text {out }}=\log _{2}\left(1+\frac{\gamma_{\mathrm{su}}}{d_{\mathrm{su}}^{\alpha}} \ln \frac{1}{(1-\varepsilon)}\right)
$$

where $d_{\text {su }}$ represents the distance between an ST and the corresponding SR.

From the data rate expressions in the second phase as shown in Equations 6 to 7, the outage probability $P_{c}^{\text {out }}$ of cooperative beamforming can be calculated as:

$$
\begin{aligned}
P_{c}^{\text {out }} & =\operatorname{Pr}\left(\frac{1}{2} \min \left(R_{p s_{1}}, R_{p s_{2}} \cdot R_{p s_{k}} \cdot, R_{p s_{M}}, R_{\mathrm{MRC}}\right)<R\right) \\
& =1-\prod_{k=1}^{M}\left(1-\operatorname{Pr}\left(R_{p s_{k}}<2 R\right)\right)\left(1-\operatorname{Pr}\left(R_{\mathrm{MRC}}<2 R\right)\right) .
\end{aligned}
$$

The two cumulative density functions $\operatorname{Pr}\left(R_{p s_{k}}<2 R\right)$ and $\operatorname{Pr}\left(R_{\mathrm{MRC}}<2 R\right)$ in the right-hand side of Equation 26 can be calculated as, respectively:

$$
\begin{aligned}
& \operatorname{Pr}\left(R_{p s_{k}}<2 R\right)=1-\exp \left(-d_{p s_{k}}^{\alpha} \cdot \frac{2^{R}-1}{\gamma_{p s_{k}}}\right), \\
& \operatorname{Pr}\left(R_{\mathrm{MRC}}<2 R\right)=F\left(2^{2 R}-1\right),
\end{aligned}
$$

where $F(z)$ can be shown to be:

$$
F(z)=\sum_{k=1}^{M+1} \frac{\left(1-\exp \left(-\lambda_{k} z\right)\right) \prod_{i=1, i \neq k}^{i=M+1} \lambda_{i}}{\prod_{i=1, i \neq k}^{i=M+1}\left(\lambda_{k}-\lambda_{i}\right)}
$$

with $z=\sum_{k=1}^{M} \gamma_{s_{k} d}\left|h_{s_{k} d}\right|^{2}+\gamma_{p}\left|h_{p}\right|^{2}, \lambda_{k}=d_{s_{k} d}^{\alpha}$, and $\lambda_{M+1}$ $=d_{p}^{\alpha} / \gamma_{p}$.

Substituting Equations 27 to 29 into 26, the outage probability $P_{c}^{\text {out }}$ is rewritten as:

$P_{c}^{\text {out }}=1-\sum_{k=1}^{M}\left(g_{s_{k} d}\right)^{d_{s_{k} d}^{\alpha}}\left(1-a\left(g_{p}\right)^{d_{p}^{\alpha}}+(1+a)\left(g_{s_{k} d}\right)^{d_{s_{k} d}^{\alpha}}\right)$, with

$$
\begin{aligned}
a & =\left(\frac{P_{s_{k} d}}{P_{p}}\left(\frac{d_{p}}{d_{s_{k}}}\right)^{\alpha}-1\right)^{-1}, \\
\mathrm{~g}_{s_{k} d} & =\exp \left(-\frac{N_{0}\left(2^{2 R}-1\right)}{P_{s_{k} d}}\right), \\
\mathrm{g}_{p} & =\exp \left(-\frac{N_{0}\left(2^{2 R}-1\right)}{P_{p}}\right) .
\end{aligned}
$$

From Equations 26 to 27 , it is obvious that $P_{\mathrm{c}}^{\text {out }}$ increases along with $R$, while $\mathrm{g}(x)$ is a decreasing function of $R$. Since $g_{s_{k} d}=g_{p} \frac{P_{p}}{P_{s_{k} d}}$, by replacing $g_{s_{k} d}$ in Equation 30 with $g_{p}$, it can be shown that $P_{c}^{\text {out }}$ is a monotonically decreasing function of $g_{p}$. Thus, by equating Equation 30 with a threshold $\varepsilon$ of outage probability, we can solve for a specific $g_{p}$, denoted with $g_{p \mid \varepsilon}$ by using the Newton method.

$$
C_{\mathrm{cb}}^{\text {out }}=\log _{2}\left(1+\gamma_{\mathrm{p}} \ln \frac{1}{g_{p \mid \varepsilon}}\right) .
$$

Eventually, the energy efficiency of the cooperative beamforming scheme, which can be used as a metric evaluating the amount of useful information bit per unitary energy, is obtained as:

$$
\eta=\frac{C_{\mathrm{cb}}^{\text {out }}}{\mathrm{P}_{N}}
$$

where $\mathrm{P}_{N}$ can be obtained from Equation 11.

A widely adopted metric for evaluating the validity for saving network energy consumption is the value of 'bits-per-Joule' for the network, i.e., the network energy consumption for unit bit of throughput, denoted with $\zeta$ and calculated as $\zeta=P_{\mathrm{N}} / R_{\mathrm{N}}$. Energy efficiency, denoted with $\eta$, is defined as ratio of outage capacity over network power consumption.

\section{Simulation results for performance evaluation}

Monte Carlo simulations are conducted to evaluate the performance of the proposed minimum power allocation algorithm utilized in cooperative beamforming for a spectrum sharing system. The mobile wireless channel is generated using an IIR filter followed by linear interpolation based on the 'sum-of-sinusoids' principle proposed by Jakes in $[39,40]$. The distance $d_{p}$ between the PT and the PR varies within the range from 0 to $1,000 \mathrm{~m}$. The distances between the STs and the PR are fixed to be $300 \mathrm{~m}$, and the distances between the STs and their corresponding SRs are set to be $200 \mathrm{~m}$. In the simulations, a ST is selected to be a relay if the distance between the PT and the ST satisfies Equation 4 and is upper-bounded by 800 $\mathrm{m}$. The parameter setting of the simulations is reported in Table 2 . 
Table 2 Parameter settings for simulation

\begin{tabular}{lc}
\hline Description & Value \\
\hline Amplifier efficiency $\rho$ & $38 \%$ \\
Circuit power $p_{C}$ & $1 \mathrm{mw}$ \\
Power for training signals $P_{\text {TR }}$ & $1 \mathrm{mw}$ \\
Bits per symbol of MS $b_{\mathrm{RD}}$ & 6 \\
Bits per symbol of CB bSR & 4 \\
Number of training symbols $N_{\text {TR }}$ & 1 \\
Number of symbols for CB $N_{\text {RD }}$ & 99 \\
Outage probability threshold $\varepsilon$ & 0.01 \\
Required QoS of PU/SU & $1 / 0.5 \mathrm{bps} / \mathrm{Hz}$ \\
Pathloss exponent $\alpha$ & 3.5 \\
PT-PR distance $d_{p}$ & $0<d_{p} \leq 1,000 \mathrm{~m}$ \\
PT-ST distance $d_{p s_{k}}$ & $d_{p s_{k}} \leq 800 \mathrm{~m}$ \\
ST-PR distance $d_{s_{k} d}$ & $d_{s_{k} d}=300 \mathrm{~m}$ \\
ST-SR distance $d_{s u}$ & $d_{s u}=200 \mathrm{~m}$ \\
Maximum transmission power $P_{\max }$ & $40 \mathrm{mw}$ \\
Noise spectral density $N_{0}$ & 0.5 \\
Number of snapshots per meter & 20 \\
SNR threshold for decoding $\theta_{1}$ & 0.01 \\
SNR of the worst link between $S T_{k}$ and PR $\theta_{2}$ & 0.0001 \\
\hline &
\end{tabular}

\subsection{Performance evaluation with the number $L_{s}$ of SUs as} a parameter

Figure $3 \mathrm{a}, \mathrm{b}$ depicts the average minimum network power consumption $P_{N}$ required for performing cooperative beamforming versus the PT-PR distance $d_{d}$ for the cases with and without considering the overhead cost $P_{\mathrm{OV}}$, respectively. The number $L_{\mathrm{S}}$ of SUs is considered as a parameter. Totally, 2,000 snapshots were simulated to calculate each point along the graph of average $P_{N}$ versus $d_{d}$. It can be observed by comparing Figure $3 \mathrm{a}, \mathrm{b}$ that in the case without considering $P_{\mathrm{OV}}$, the average minimum network power consumption $P_{N}$ decreases dramatically when $L_{s}$ increases, while in the situation where $P_{\mathrm{OV}}$ is taken into account, the average $P_{N}$ graphs appear to be less deviated from each other when $L_{s}$ changes. This observation indicates that the energy costs for relay scheduling and information sharing can increase along with the number of the SUs involved. The advantage of cooperative beamforming is counteracted by the increasing of overheads to some extent.

Figure 4 illustrates the variation of $\zeta$, i.e., the network energy consumption per unit throughput versus the PTPR distance $d_{d}$ with the overhead consumption $P_{\mathrm{OV}}$ considered. The number $L_{s}$ of SUs is also taken as a parameter in the simulations. It can be observed that $\zeta$ decreases significantly with the growth of the SU number. This is due to the fact that the network throughput $R_{N}$ increases enormously along with $L_{s}$, and meanwhile, no evident

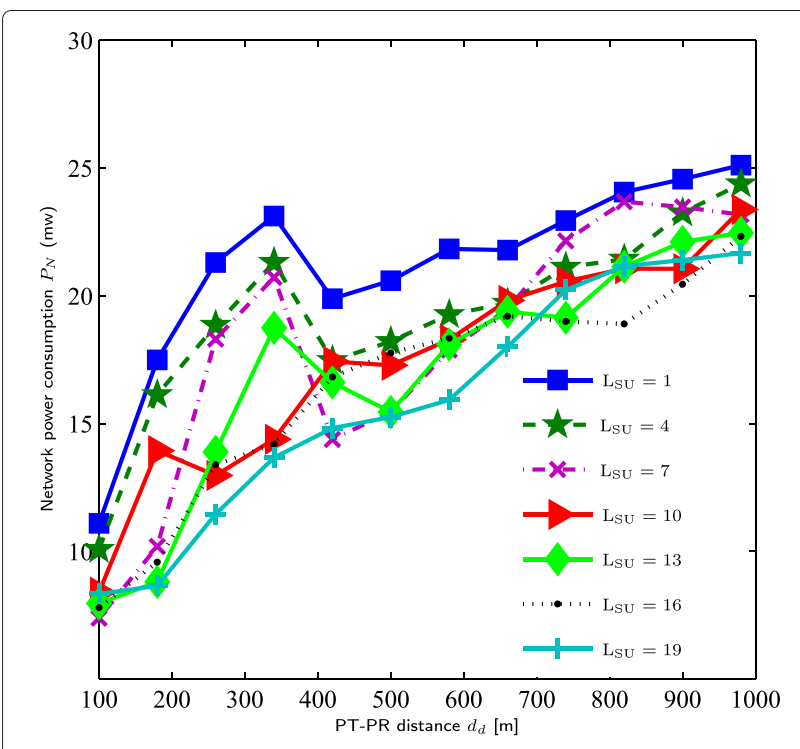

(a) Overheads not considered

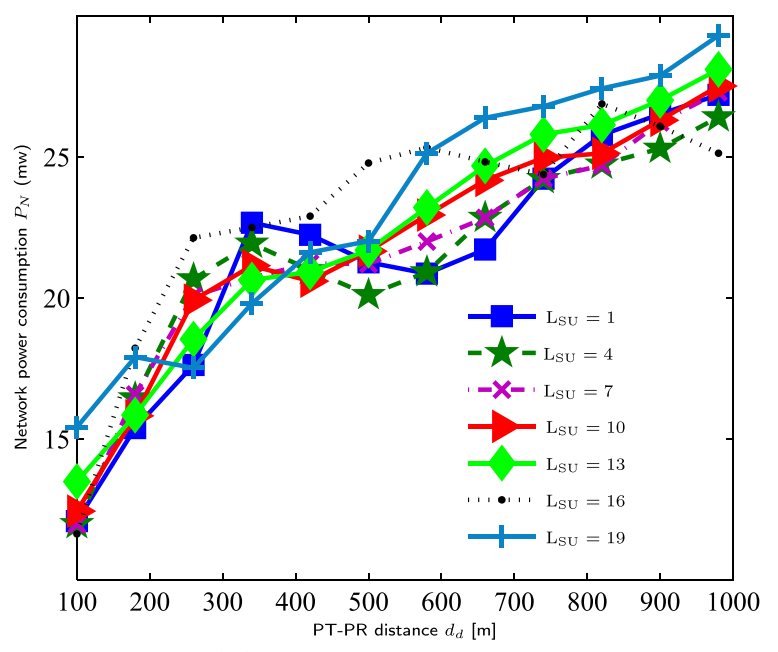

(b) Overheads considered

Figure 3 Average minimum power consumption versus the PT-PR distance $d_{d}$ for different SU numbers with/without overhead considered. (a) Overheads not considered. (b) Overheads considered.

increase of network power consumption is observed when $L_{s}$ grows when the overhead and scheduling consumption is considered.

The variation of the energy efficiency $\eta$ for the cooperative beamforming scheme when $L_{s}$ changes is illustrated in Figure 5. The overhead consumption has been considered in the simulations. Every scatter plot in Figure 5 is calculated by averaging the energy efficiency obtained with various $d_{d}$ from 2,000 snapshots. It is obvious from Figure 5 that the average $\eta$ grows linearly with the increasing number of SUs. A linear curve with a slope of 10 


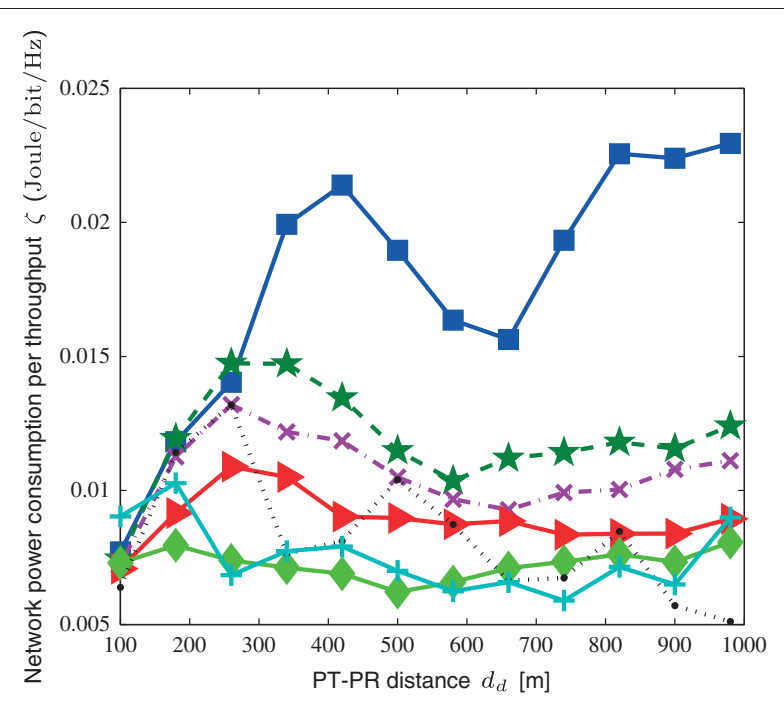

Figure 4 Network power consumption per unit throughput $\zeta$ versus the PT-PR distance $d_{d}$ for different SU numbers with overhead considered. The legend shown in Figure 3 also applies here.

$\mathrm{bit} / \mathrm{s} / \mathrm{J} / \mathrm{SU}$ is found fitting well with the simulation results. The observation of more bits being transmitted when $L_{s}$ increases is due to the joint effect that the enhanced capacity of the cooperative beamforming system for larger $L_{s}$, and at the same time, the network power consumption in the cooperative beamforming phase is reduced with more SUs involved.

\subsection{Comparison among three communication schemes}

Figure 6 compares the network power consumption for three cases, i.e., best relay selection with the number of SUs $L_{s}=6$, non-cooperation scheme with single relay, and the cooperative beamforming with $L_{s}=6$.

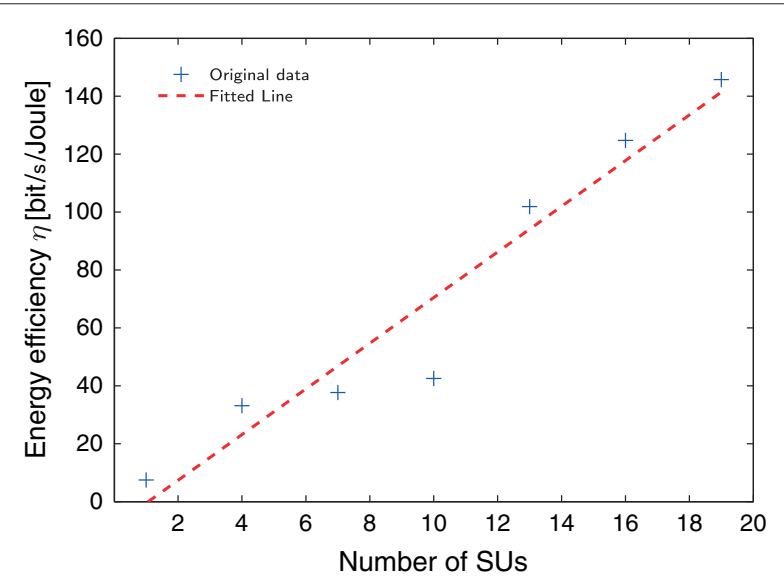

Figure 5 Energy efficiency versus SU number with overhead considered.

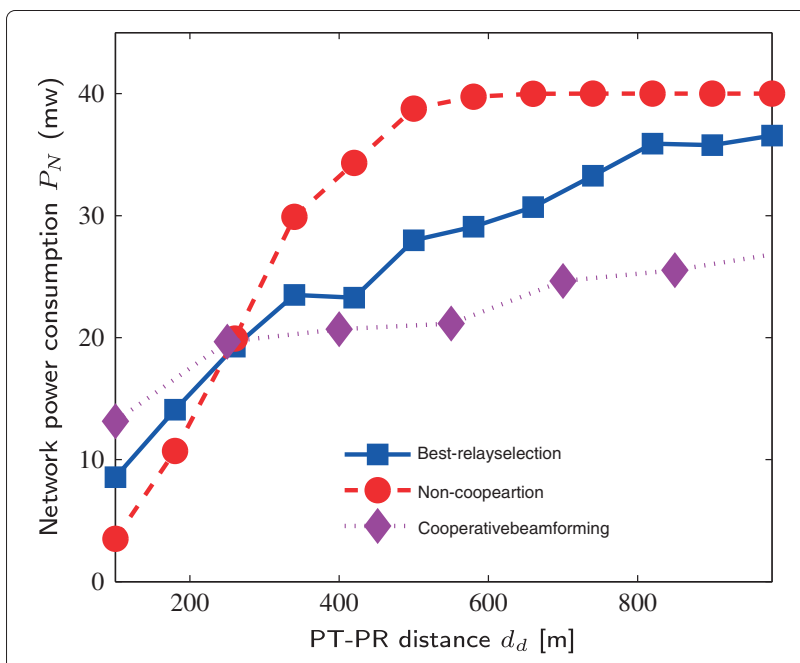

Figure 6 Average minimum power consumption versus the PT-PR distance $d_{d}$ for three communication schemes with the number of SUs $L_{s}=6$.

In the best relay selection algorithm, the optimal relay is selected with the criterium of maximum energy efficiency from $M$ candidate relays [35]. The threshold of QoS requirement of the PU and the SUs are set as respectively, 1 and $0.5 \mathrm{bps} / \mathrm{Hz}$ in the simulated communication schemes. It can be observed that the proposed cooperative beamforming scheme outperforms the other two, and the best-relay selection algorithm exhibits the intermediate performance. This is the rationale as the implementation of the relay selection process may deteriorate the performance in some cases when compared with the cooperative beamforming scheme. Furthermore, for a fixed number of SUs, without significant benefits from the energy minimization, the best relay selection scheme consumes the similar amount of energy for scheduling and of circuit power as the cooperative beamforming does.

Figure 7a,b illustrates the throughput $R_{d}$ of the direct transmission, the network throughput $R_{N}$, the average PU's throughput $R_{p}$ in the first and second phases, and the average SU's throughput $\bar{R}_{S}=\frac{1}{M} \sum_{k=1}^{M} R_{s u_{k}}$, versus the PT-PR distance $d_{d}$ for the best-relay-selection scheme and the proposed cooperative beamforming scheme, respectively. The number $L_{s}$ of SUs equals 6 in the simulations. It can be found from Figure 7a,b that the direct transmission provides less $R_{d}$ for increasing $d_{d}$, and on the contrary, the PU's throughput $R_{P}$ and the network throughput $R_{N}$ with cooperative beamforming increase along with $d_{d}$. The throughput of SUs remains a constant level irrespective of $d_{d}$ as expected. These results reveal that the relay and the cooperative beamforming systems can provide higher throughput than the direct transmission. It can be furthermore observed by comparing Figure $7 \mathrm{a}, \mathrm{b}$ that 


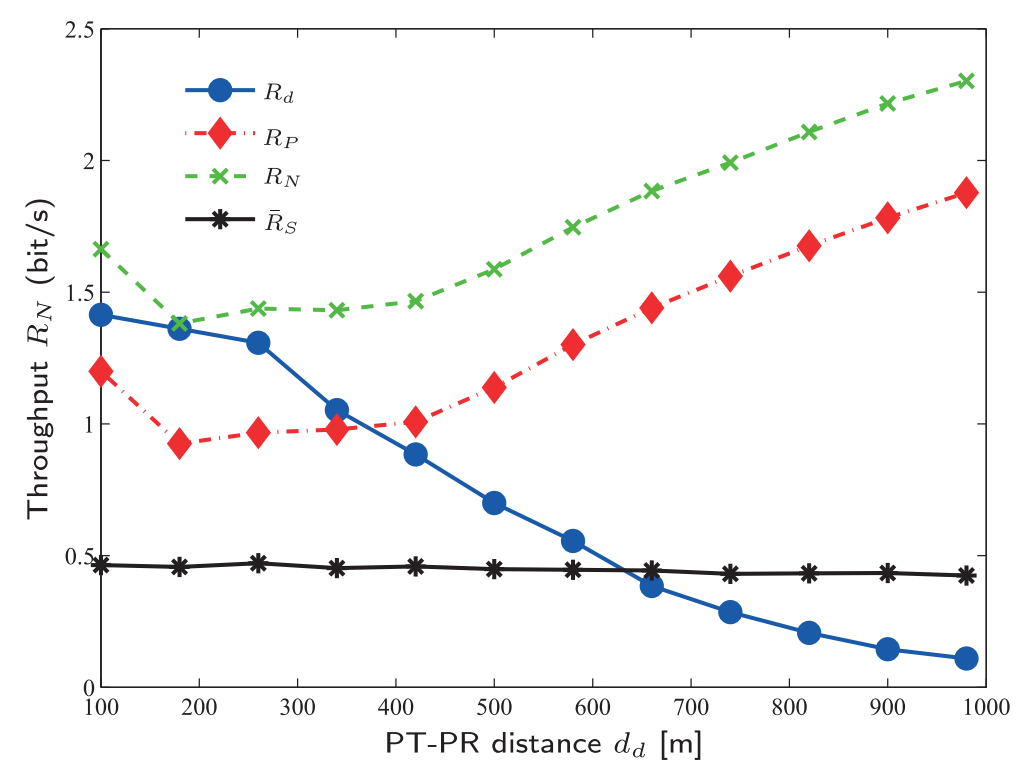

(a) Relay selection

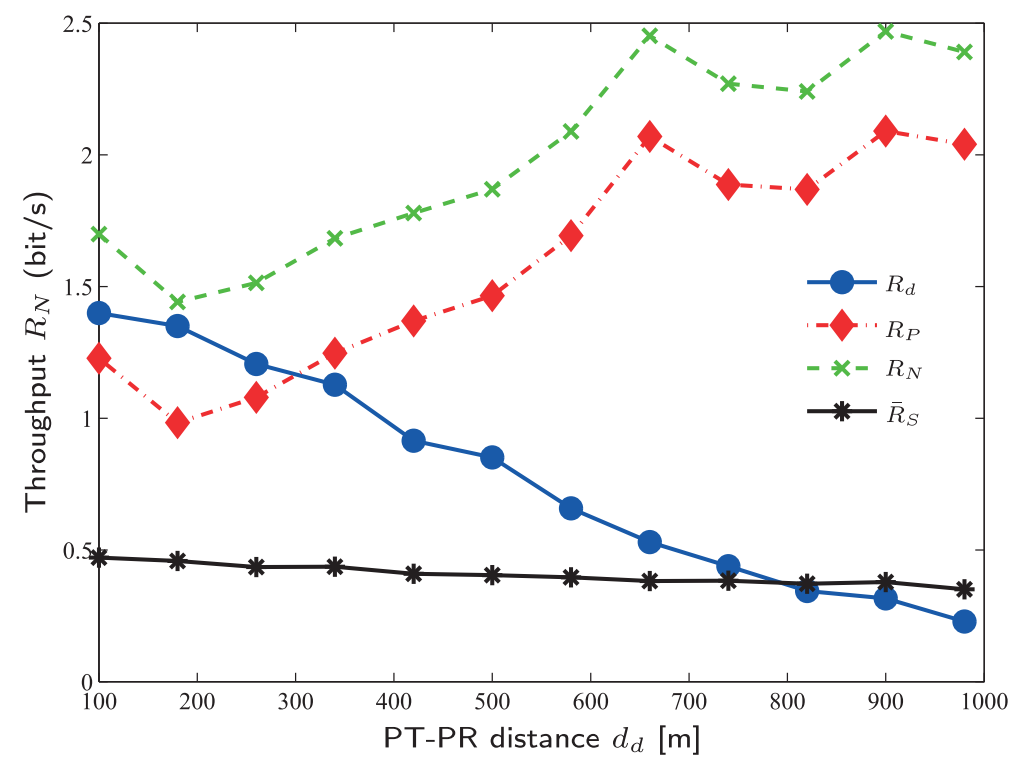

(b) Cooperative beamforming

Figure 7 Throughput versus the PT-PR distance $d_{d}$ for relay-selection and cooperative beamforming schemes with the number of SUs $L_{s}=6$. (a) relay selection. (b) Cooperative beamforming.

the proposed cooperative beamforming scheme exhibits throughput graphs similar to those obtained for the best relay scheme. However, since the network consumption is less for the cooperative beamforming than for the best relay scheme as illustrated in Figure 6, it is a natural conjecture that the energy efficiency, i.e., the network power consumption per unit throughput would be less in the case of cooperative beamforming than of the best relay. This is confirmed by the results shown in Figure 8 where the 'Joule-per-bit' for the cooperative beamforming is nearly a half of that observed for best relay selection with large $d_{\mathrm{d}}$.

Figure 9 compares the network energy efficiency $\eta$ versus the PT-PR distance $d_{d}$ for the best relay selection, non-cooperative and cooperative beamforming schemes with $L_{s}=6$. It can be observed that the network energy efficiency of cooperative beamforming remains constant for different values of $d_{d}$ and is the highest among all three schemes considered. Furthermore, the graph of the energy efficiency of the best relay scheme exhibits 


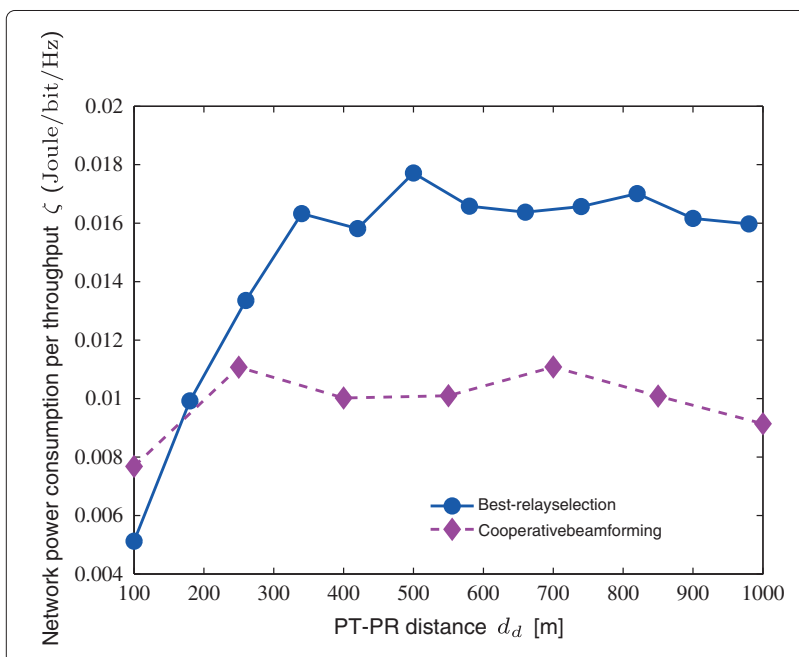

Figure 8 Network power consumption per unit throughput $\zeta$ versus the PT-PR distance $d_{d}$ of two cases with $L_{s}=6$.

a concave shape with the maximum obtained with the distance equal to $500 \mathrm{~m}$. This is consistent with the analysis shown in $[41,42]$ that the network energy efficiency is concave with respect to the transmission power which monotonically increases along with the distance. Furthermore, in the case with short distances, the outage capacity is small, and a certain level of total power consumption still remains. Consequently, the energy efficiency becomes extremely low. In cases where the distance is sufficiently large, the outage capacity becomes saturated. In such case, since the power consumption keeps increasing, the energy efficiency decreases and converges to zero for large $d_{d}$.

\subsection{Performance evaluation in vehicular scenarios}

The performance of the cooperative beamforming scheme with proposed power allocation strategy is also evaluated

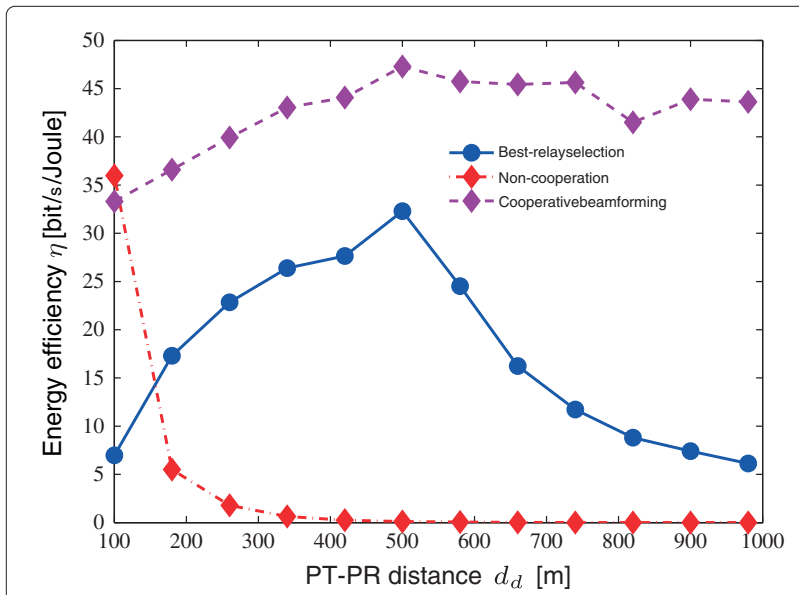

Figure 9 Network energy efficiency versus the PT-PR distance $d_{\mathrm{d}}$ of three cases with $L_{s}=6$. by simulations in time-variant cases where the nodes in the network move in high speeds. In the simulations, the speeds of the PU and of the SUs are set to be identical and represented with $v$. The channel coefficients among the nodes are generated by using a tap-delay-line (TDL) model with six discrete paths. The number $L_{\mathrm{s}}$ of SUs is fixed to be 5 .

Figure 10 depicts the network power consumption $P_{N}$ versus the PT-PR distance $d_{d}$ for moving speed $v=5$, 50 , and $100 \mathrm{~km} / \mathrm{h}$. It can be observed from Figure 10 that the graph of $P_{N}$ versus $d_{d}$ observed for $v=100$ $\mathrm{km} / \mathrm{h}$ is only slightly above those obtained with less $v$. This indicates that the network power consumption for the cooperative beamforming is insensible to the speed of the nodes involved. Figures 11, 12, and 13 depict, respectively, the network throughput $R_{N}$, the energy consumption per unit throughput, and the energy efficiency $\eta$ versus the PT-PR distance $d_{d}$ for different $v$. From these figures, it can be observed that the performance of the cooperative beamforming scheme with proposed power allocation only slightly degrades when the speed of nodes increases. These results demonstrate the robustness of the proposed scheme against the time variability of the channels and the applicability of the scheme in vehicular scenarios.

\section{Conclusions}

In this paper, a novel power allocation scheme has been proposed for energy-efficient mobile cooperative communication with spectrum sharing. In the system, SUs act collaboratively as beamforming relays and help the PU in data transmission in reward of transmitting their own information. The optimal power allocation derived solves a convex optimization problem of minimizing network

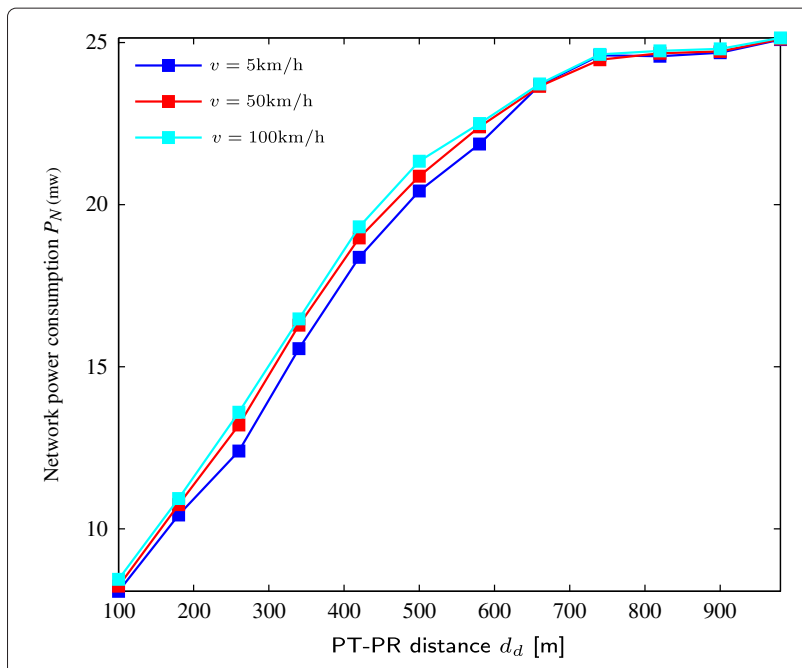

Figure 10 Network power consumption $P_{N}$ versus the PT-PR distance $d_{d}$ with UE speed as a parameter. 


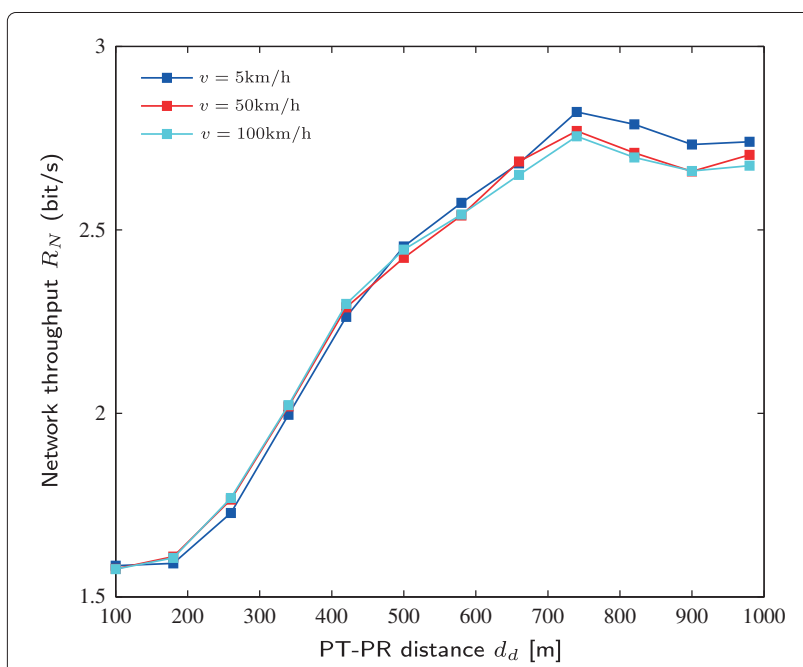

Figure 11 Network throughput $R_{N}$ versus the PT-PR distance $d_{d}$ with UE speed as a parameter.

energy consumption under the constraint of the guaranteed quality of service for both PU and SUs. Analytical expressions were derived based on the resultant optimal power allocation for outage capacity of the system. Numerical simulations have been conducted for evaluating the performance of the proposed scheme. The results demonstrated that the network power consumption exhibited different behaviors depending on whether the overhead power consumption is considered. By taking into account the overhead power consumption, circuit power, and message sharing energy costs, the cooperative beamforming cognitive network together with the proposed power allocation method performs better when

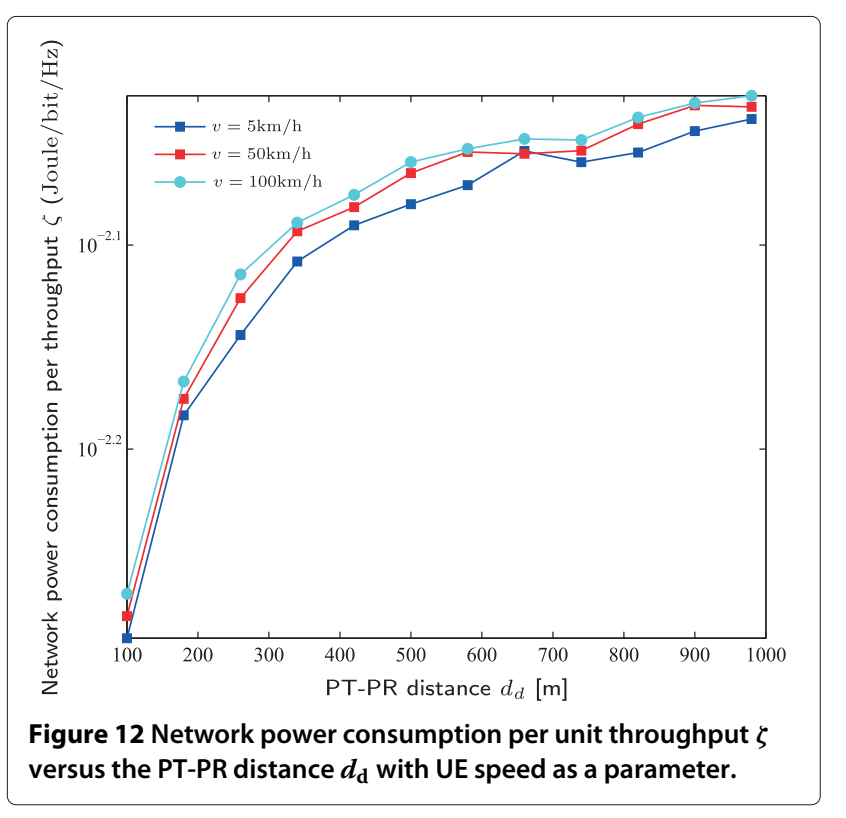

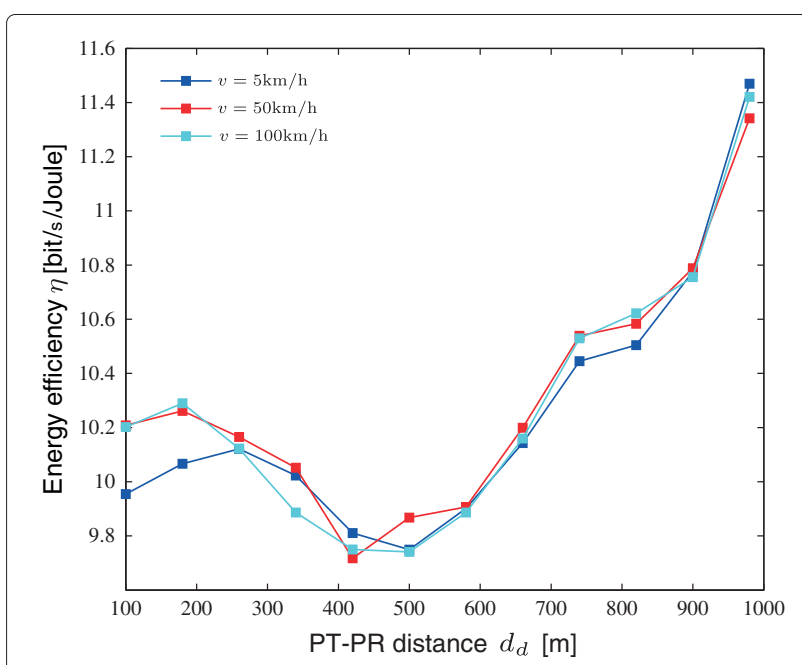

Figure 13 Network energy efficiency $\eta$ versus the PT-PR distance $d_{d}$ with UE speed as a parameter.

more SUs are involved, in terms of enhanced energy efficiency and reduced power consumption per unit throughput. Furthermore, simulations demonstrated that the proposed cooperative beamforming scheme is superior to the conventional best relay selection scheme in the aspects of network power consumption, power consumption per unit throughput, and energy efficiency. In addition, the cooperative beamforming scheme with the optimal power allocation proposed here exhibited stable performance in the time-variant scenarios where both the PU and SUs move in high speeds.

\section{Appendix 1}

\section{Convexity analysis of the optimization problem in Equations 15 to 18}

A convex optimization problem is one in which the objective and constraint functions are convex [37]. In general, a continuous and twice differentiable function is concave, if and only if its Hessian matrix is negative semi-definite. On the contrary, if the Hessian matrix is positively semidefinite, the function is convex. Now, we discuss the convexity of the two inequality constraints (16) and (17), i.e.,

$$
\begin{aligned}
& \beta_{k} Q_{p}-\frac{1}{2} \log _{2}\left(1+\frac{P_{p}\left|h_{p}\right|^{2}}{N_{o}}\right)-\frac{1}{2} \log _{2}\left(1+\frac{P_{s_{k} d}\left|h_{s_{k} d}\right|^{2}}{N_{o}}\right) \leq 0 \text { and } \\
& Q_{s}-\frac{\beta_{k}-1}{\beta_{k}} \log _{2}\left(1+\frac{P_{\text {suk }}\left|h_{s u_{k}}\right|^{2}}{N_{0}}\right) \leq 0 .
\end{aligned}
$$

Denoting $f\left(P_{p}, P_{s_{k}} d, \beta_{k}\right)=\beta_{k} Q_{p}-\frac{1}{2} \log _{2}\left(1+\frac{P_{p}\left|h_{p}\right|^{2}}{N_{o}}\right)-$ $\frac{1}{2} \log _{2}\left(1+\frac{P_{s_{k} d}\left|h_{s_{k}} d\right|^{2}}{N_{o}}\right)$, we can compute the Hessian matrix of $f\left(P_{p}, P_{s_{k}}, \beta_{k}\right)$ as: 


$$
\begin{aligned}
H_{f} & =\left(\begin{array}{ccc}
\frac{\partial^{2} f\left(P_{p}, P_{s_{k} d}, \beta_{k}\right)}{\partial P_{p}{ }^{2}} & \frac{\partial^{2} f\left(P_{p}, P_{s_{k} d}, \beta_{k}\right)}{\partial P_{p} \partial P_{s_{k}} d} & \frac{\partial^{2} f\left(P_{p}, P_{s_{k} d}, \beta_{k}\right)}{\partial P_{p} \partial \beta_{k}} \\
\frac{\partial^{2} f\left(P_{p}, P_{s_{k} d}, \beta_{k}\right)}{\partial P_{s_{k} d} P_{p}} & \frac{\partial^{2} f\left(P_{p}, P_{s_{k} d}, \beta_{k}\right)}{\partial P_{s_{s} d^{2}}} & \frac{\partial^{2} f\left(P_{p}, P_{s_{k} d}, \beta_{k}\right)}{\partial P_{s_{k} d} \partial \beta_{k}} \\
\frac{\partial^{2} f\left(P_{p}, P_{s_{k} d}, \beta_{k}\right)}{\partial \beta_{k} \partial P_{p}} & \frac{\partial^{2} f\left(P_{p}, P_{s_{k} d}, \beta_{k}\right)}{\partial \beta_{k} \partial P_{s_{k} d}} & \frac{\partial^{2} f\left(P_{p}, P_{s_{k} d}, \beta_{k}\right)}{\partial \beta_{k}{ }^{2}}
\end{array}\right) \\
= & \left(\begin{array}{ccc}
\frac{1}{2}\left(\frac{\left|h_{p}\right|^{2} \ln 2}{N_{0}+P_{p}\left|h_{p}\right|^{2}}\right)^{2} & 0 & 0 \\
0 & \frac{1}{2}\left(\frac{\left|h_{s_{k} d}\right|^{2} \ln 2}{N_{0}+\left.\left.P_{s_{k} d}\right|_{s_{k} d}\right|^{2}}\right)^{2} & 0 \\
0 & 0 & 0
\end{array}\right) .
\end{aligned}
$$

Thus, the eigenvalues of $H_{f}$ are all above or equal to zero, which shows that $H_{f}$ is a positive semi-definite matrix, an implication that $f\left(P_{p}, P_{s_{k} d}, \beta_{k}\right)$ is a convex function and Equation 16 is a convex constraint.

Similarly, denoting $g\left(P_{s u_{k}}, \beta_{k}\right)=Q_{s}-\frac{\beta_{k}-1}{\beta_{k}} \log _{2}$ $\left(1+\frac{P_{s u_{k}}\left|h_{s u_{k}}\right|^{2}}{N_{0}}\right)$, we can obtain the Hessian matrix of $g\left(P_{s u_{k}}, \beta_{k}\right)$ as:

$H_{g}=\left(\begin{array}{cc}\frac{\beta_{k}-1}{\beta_{k}} \frac{\left|h_{s u_{k}}\right|^{4}(\ln 2)^{2}}{\left[N_{0}+P_{s u_{k}}\left|h_{s u_{k}}\right|^{2}\right]^{2}} & \frac{1}{\beta_{k}{ }^{2}} \frac{\left|h_{s u_{k}}\right|^{2} \ln 2}{\left[N_{0}+P_{s u_{k}}\left|h_{s u_{k}}\right|^{2}\right]} \\ \frac{1}{\beta_{k}^{2}} \frac{\left|h_{s u_{k}}\right|^{2} \ln 2}{\left[N_{0}+P_{s u_{k}}\left|h_{s u_{k}}\right|^{2}\right]} & \frac{2}{\beta_{k}{ }^{3}} \log _{2}\left(1+\frac{P_{s u_{k}}\left|h_{s u_{k}}\right|^{2}}{N_{0}}\right)\end{array}\right)$.

Thus, the determinants of all leading principal minors of the $H_{g}$ are given by:

$\left|H_{g}^{1}\right|=\frac{\beta_{k}-1}{\beta_{k}} \frac{\left|h_{s u_{k}}\right|^{4}(\ln 2)^{2}}{\left[N_{0}+P_{s u_{k}}\left|h_{s u_{k}}\right|^{2}\right]^{2}}>0$
$\left|H_{g}^{2}\right|=\left[\frac{1}{\beta_{k}{ }^{4}} \frac{\left|h_{s u_{k}}\right|^{4}(\ln 2)^{2}}{\left[N_{0}+P_{s u_{k}}\left|h_{s u_{k}}\right|^{2}\right]^{2}}\right]\left[2\left(\beta_{k}-1\right) \log _{2}\left(1+\frac{P_{s u_{k}}\left|h_{s u_{k}}\right|^{2}}{N_{0}}\right)-1\right]$.

From Equation 17, it can be shown that:

$$
\left[2\left(\beta_{k}-1\right) \log _{2}\left(1+\frac{P_{s u_{k}}\left|h_{s u_{k}}\right|^{2}}{N_{0}}\right)-1\right] \geq 2 \beta_{k} Q_{s}-1 .
$$

Thus, as long as the requirement of $2 \beta_{k} Q_{s}-1 \geq 0$, i.e., $Q_{s} \geq 0.5$ is satisfied, it is easy to show that Equation 17 is a convex constraint.

Furthermore, denoting the objective function in Equation $15 z\left(P_{p}, P_{s_{k}}, P_{s u_{k}}, \beta_{k}\right)=\frac{1}{2 \beta_{k}}\left(P_{p}+P_{s_{k}} d\right)+$ $\frac{\beta_{k}-1}{\beta_{k}} P_{s u_{k}}$, the Hessian matrix of $z\left(P_{p}, P_{s_{k}}, P_{s u_{k}}, \beta_{k}\right)$ can be shown as:

$$
H_{z}=\left(\begin{array}{cccc}
0 & 0 & 0 & -\frac{1}{2 \beta_{k}{ }^{2}} \\
0 & 0 & 0 & -\frac{1}{2 \beta_{k^{2}}} \\
0 & 0 & 0 & -\frac{1}{\beta_{k}{ }^{2}} \\
-\frac{1}{2 \beta_{k}{ }^{2}} & -\frac{1}{2 \beta_{k}{ }^{2}} & -\frac{1}{\beta_{k}{ }^{2}} & \frac{1}{\beta_{k}{ }^{3}}\left[P_{p}+P_{s_{k} d}-2 P_{s u_{k}}\right]
\end{array}\right) .
$$

It can be seen that all leading principal minors of $H_{z}$ are equal to zero matrixes. Thus, $z\left(P_{p}, P_{s_{k} d}, P_{s u_{k}}, \beta_{k}\right)$ is a convex function in terms of $\left(P_{p}, P_{s_{k} d}, P_{s u_{k}}, \beta_{k}\right)$ and the objective function in Equation 15 is convex.

In addition, the constraint (18) is a linear function concerning to $\beta_{k}$. Thus, the Hessian matrixes is zero matrix, indicating the convexity of the constraint (18).

Based on these analyses, we conclude that the formulated optimization problem in Equations 15 to 18 is convex, under the condition that QoS of SU is larger than or equal to 0.5 .

\section{Appendix 2}

Solution of the optimization problem in Equations 15 to 18 As Equations 15 to 18 are a convex optimization problem with differentiable objective and constraint, the KKT conditions are necessary and sufficient for the optimality. Furthermore, $T$ is normalized to be 1 without loss of generality. By adding Lagrange multipliers, i.e., $\lambda, \mu, v$, according to the constraints of Equations 16 to 18, the Lagrange dual function can be formulated as:

$$
\begin{aligned}
L\left(P, \beta_{k}, \lambda, \mu, \nu\right)= & \frac{1}{2 \beta_{k}}\left(P_{p}+P_{s_{k} d}\right)+\frac{\beta_{k}-1}{\beta_{k}} P_{s u_{k}} \\
& +\lambda\left(\beta_{k} Q_{p}-\frac{1}{2} \log _{2}\left(1+\frac{P_{p}\left|h_{p}\right|^{2}}{N_{o}}\right)-\frac{1}{2} \log _{2}\left(1+\frac{P_{s_{k} d}\left|h_{s_{k} d}\right|^{2}}{N_{o}}\right)\right) \\
& +\mu\left(Q_{s}-\frac{\beta_{k}-1}{\beta_{k}} \log _{2}\left(1+\frac{P_{s u_{k}}\left|h_{s u_{k}}\right|^{2}}{N_{0}}\right)\right)+v\left(1-\beta_{k}\right) .
\end{aligned}
$$

By solving the following equations simultaneously:

$$
\begin{aligned}
& \frac{\partial L\left(P, \beta_{k}, \lambda, \mu, v\right)}{\partial P_{p}}=0, \\
& \frac{\partial L\left(P, \beta_{k}, \lambda, \mu, v\right)}{\partial P_{s_{k} d}}=0, \\
& \frac{\partial L\left(P, \beta_{k}, \lambda, \mu, v\right)}{\partial P_{s u_{k}}}=0, \\
& \frac{\partial L\left(P, \beta_{k}, \lambda, \mu, v\right)}{\partial \beta_{k}}=0, \\
& \lambda\left(\beta_{k} Q_{p}-\frac{1}{2} \log _{2}\left(1+\frac{P_{p}\left|h_{p}\right|^{2}}{N_{o}}\right)-\frac{1}{2} \log _{2}\left(1+\frac{P_{s_{k} d}\left|h_{s_{k} d}\right|^{2}}{N_{o}}\right)\right)=0, \\
& \mu\left(Q_{s}-\frac{\beta_{k}-1}{\beta_{k}} \log _{2}\left(1+\frac{P_{s u_{k}}\left|h_{s u_{k}}\right|^{2}}{N_{0}}\right)\right)=0, \\
& v\left(1-\beta_{k}\right)=0, \\
& \beta_{k} Q_{p}-\frac{1}{2} \log _{2}\left(1+\frac{P_{p}\left|h_{p}\right|^{2}}{N_{o}}\right)-\frac{1}{2} \log _{2}\left(1+\frac{P_{s_{k} d}\left|h_{s_{k} d}\right|^{2}}{N_{o}}\right) \geq 0, \\
& Q_{s}-\frac{\beta_{k}-1}{\beta_{k}} \log _{2}\left(1+\frac{P_{s u_{k}}\left|h_{s u_{k}}\right|^{2}}{N_{0}}\right) \geq 0, \\
& 1-\beta_{k} \geq 0,
\end{aligned}
$$


we obtain the optimal point in terms of the powers:

$$
\begin{aligned}
& P_{p}=N_{o}\left(\frac{2^{\beta_{k} Q_{p}}}{\left|h_{p}\right|\left|h_{s_{k} d}\right|}-\frac{1}{\left|h_{p}\right|^{2}}\right), \\
& P_{s_{k} d}=N_{o}\left(\frac{2^{\beta_{k} Q_{p}}}{\left|h_{p}\right|\left|h_{s_{k} d}\right|}-\frac{1}{\left|h_{s_{k} d}\right|^{2}}\right), \\
& P_{s u_{k}}=\frac{N_{o}}{\left|h_{s u_{k}}\right|^{2}}\left(2^{\frac{\beta_{k} Q_{s}}{\beta_{k}-1}}-1\right) .
\end{aligned}
$$

Once these optimal transmission powers are known, the optimal $\beta_{k}$ can be calculated by solving the function:

$$
\begin{gathered}
\frac{2^{\beta_{k} Q_{p}}}{\left|h_{s_{k} d}\right|\left|h_{p}\right|}\left(Q_{p} \beta_{k}-1\right)-\frac{\beta_{k} Q_{s} 2^{\frac{\beta_{k} Q_{s}}{\beta_{k}-1}}}{\left|h_{s u_{k}}\right|^{2}\left(\beta_{k}-1\right)} \\
+\frac{2^{\frac{\beta_{k} Q_{s}}{\beta_{k}-1}}-1}{\left|h_{s u_{k}}\right|^{2}}+\frac{1}{2\left|h_{p}\right|^{2}}+\frac{1}{2\left|h_{s_{k}}\right|^{2}}=0 .
\end{gathered}
$$

Denoting the left-hand side of Equation 35 as $f\left(\beta_{k}\right)$ and taking the derivative of $f\left(\beta_{k}\right)$ with respect to $\beta_{k}$ as follows:

$$
f^{\prime}\left(\beta_{k}\right)=\frac{Q_{p}{ }^{2} \beta_{k} 2^{\beta_{k} Q_{p}}}{\left|h_{s_{k} d}\right|\left|h_{p}\right|}+\frac{Q_{s}{ }^{2} \beta_{k} 2^{\frac{\beta_{k} Q_{s}}{\beta_{k}-1}}}{\left|h_{s u_{k}}\right|^{2}\left(\beta_{k}-1\right)^{3}},
$$

it is clear that Equation 36 is always larger than zero, and hence, $f\left(\beta_{k}\right)$ increases monotonically along with $\beta_{k}$. Thus, the optimal $\beta_{k}$ can be determined by applying the Newton's iterative method as described in [38]. Given an initial $\beta_{k}$ as $\beta_{k}(0)$, the following process is repeated:

$$
\beta_{k}(n+1)=\beta_{k}(n)-\frac{f\left(\beta_{k}(n)\right)}{f^{\prime}\left(\beta_{k}(n)\right)}
$$

until a sufficiently accurate value is reached.

\section{Competing interests}

The authors declare that they have no competing interests.

\section{Authors' contributions}

$\mathrm{CL}$ carried out the generic studies, performed the simulation platform, and drafted the manuscript. XY gave guidance in the implementation of the simulation software and modification of the contribution. SRB conceived of the study, participated in its design and coordination, and helped to draft the manuscript. MGL offered some help in the conceiving of the research. All authors read and approved the final manuscript.

\section{Acknowledgements}

This work has been done as a collaboration between research institutions members of European COST IC1004 Action on Cooperative Radio Communications for Green Smart Environments. It has been jointly supported by the Spanish Ministry of Science under the project TEC2011-27723-C02-01, the Sino-Spanish Campus between Tongji University and UPC, the project 'System design and demo-construction for cooperative networks of high-efficiency $4 \mathrm{G}$ wireless communications in urban hot-spot environments' granted by the Science and Technology Commission of Shanghai Municipality, China, and the NSFC general project with Grant No. 61471268. Thanks for the technical guidance of Prof. Joan Olmos from the University of Polytechnic in Catalunya.

\begin{abstract}
Author details
${ }^{1}$ College of Electronics and Information Engineering, Tongji University, 4800 Caoan Road, Shanghai, 201804, China. ${ }^{2}$ Department of Signal Theory and Communications, University of Polytechnic in Catalunya, Building C4 C. Esteve Terradas, 7 Castelldefels, 08860, Spain.
\end{abstract}

Received: 1 September 2014 Accepted: 2 March 2015

Published online: 13 March 2015

\section{References}

1. J Andrews, S Buzzi, W Choi, S Hanly, A Lozano, A Soong, J Zhang, What will 5 G be. IEEE J. Sel. Areas Commun. 32, 1065-1082 (2014)

2. I C-Lin, C Rowell, S Han, Z Xu, G Li, Z Pan, Toward green and soft: a $5 \mathrm{G}$ perspective. IEEE Commun. Mag. 52, 66-73 (2014)

3. P Demestichas, A Georgakopoulos, D Karvounas, K Tsagkaris, V Stavroulaki, J Lu, C Xiong, J Yao, 5G on the horizon: key challenges for the radio-access network. IEEE Veh. Technol. Mag. 8, 47-53 (2013)

4. SChen, J Zhao, The requirements, challenges, and technologies for $5 \mathrm{G}$ of terrestrial mobile telecommunication. IEEE Commun. Mag. 52, 36-43 (2014)

5. Y Yu, Y Chen, J Tang, D So, Z Xu, C-L I, P Ferrand, J-M Gorce, C-H Tang, P-R Li, K-T Feng, L-C Wang, K Borner, L Thiele, Green transmission technologies for balancing the energy efficiency and spectrum efficiency trade-off. IEEE Commun. Mag. 52, 112-120 (2014)

6. X Hong, J Wang, C-X Wang, J Shi, Cognitive radio in 5G: a perspective on energy-spectral efficiency trade-off. IEEE Commun. Mag. 52, 46-53 (2014)

7. W Zhuang, M Ismail, Cooperation in wireless communication networks. IEEE Wireless Commun. 19, 10-20 (2012)

8. A Papadogiannis, D Gesbert, E Hardouin, in Proceedings of IEEE International Conference on Communications (ICC). A dynamic clustering approach in wireless networks with multi-cell cooperative processing (IEEE Beijing, China, 2008), pp. 4033-4037

9. Z Niu, Y Wu, J Gong, Z Yang, Cell zooming for cost-efficient green cellular networks. IEEE Commun. Mag. 48, 74-79 (2010)

10. J Laneman, D Tse, GW Wornell, Cooperative diversity in wireless networks: efficient protocols and outage behavior. IEEE Trans. Inf. Theory. 50, 3062-3080 (2004)

11. A Bletsas, A Khisti, D Reed, A Lippman, A simple cooperative diversity method based on network path selection. IEEE J. Selec. Areas Commun. 24, 659-672 (2006)

12. YJ, H Jafarkhani, Network beamforming using relays with perfect channe information. IEEE Trans. Inf. Theory. 55, 2499-2517 (2009)

13. C Lo, S Vishwanath, R Heath, Relay subset selection in wireless networks using partial decode-and-forward transmission. IEEE Trans. Veh. Technol. 58, 692-704 (2009)

14. B Hamdaoui, T Alshammari, M Guizani, Exploiting 4G mobile user cooperation for energy conservation: challenges and opportunities. IEEE Wireless Commun. 20, 62-67 (2013)

15. Z Zhou, S Zhou, S Cui, J-H Cui, in Proceedings of IEEE Military Communications Conference (MILCOM). Energy-efficient cooperative communication in clustered wireless sensor networks (IEEE Washington, USA, 2006), pp. 1-7

16. H Shan, W Zhuang, Z Wang, Distributed cooperative MAC for multihop wireless networks. IEEE Commun. Mag. 47, 126-133 (2009)

17. D Yang, X Fang, G Xue, Game theory in cooperative communications. IEEE Wireless Commun. Mag. 19, 44-49 (2012)

18. G R Bella, G Costantino, Evaluating the device reputation through full observation in MANETs. J. Inf. Ass. Sec. 4, 458-465 (2009)

19. D Liu, W Wang, W Guo, 'Green' cooperative spectrum sharing communication. IEEE Commun. Letters. 17, 459-462 (2013)

20. C Comaniciu, NB Mandayam, H Poor, J-M Gorce, An auctioning mechanism for green radio. J. Commun. Netw-s. Kor. 12, 114-121 (2010)

21. J Furthmuller, O Waldhorst, in Proceedings of Eighth International Conference on Wireless On-Demand Network Systems and Services (WONS). Energy-aware resource sharing with mobile devices (IEEE Bardonecchia Italy, 2011), pp. 52-59

22. K Woradit, T Quek, W Suwansantisuk, H Wymeersch, L Wuttisittikulkij, M Win, Outage behavior of selective relaying schemes. IEEE Trans. Wireless. Commun. 8, 3890-3895 (2009)

23. E Larsson, Y Cao, Collaborative transmit diversity with adaptive radio resource and power allocation. IEEE Commun. Lett. 9, 511-513 (2005) 
24. Y Cao, B Vojcic, M Souryal, in Proceedings of IEEE Vehicular Technology Conference (VTC2004-Fal). User-cooperative transmission with channel feedback in slow fading environmentA (IEEE Los Angeles, USA, 2004), pp. 2063-2067

25. G Lim, J Cimini, LJ, Energy-efficient cooperative beamforming in clustered wireless networks. IEEE Trans. Wireless. Commun. 12, 1376-1385 (2013)

26. C Isheden, Z Chong, E Jorswieck, G Fettweis, Framework for link-level energy efficiency optimization with informed transmitter. IEEE Trans. Wireless. Commun. 11, 2946-2957 (2012)

27. D To, T To, J Choi, in Proceedings of IEEE Vehicular Technology Conference (VTC Spring). Energy efficient distributed beamforming with sensor selection in wireless sensor networks (IEEE Yokohama, Japan, 2012), pp. 1-5

28. $Y$ Zhang, H Dai, Energy-efficiency and transmission strategy selection in cooperative wireless sensor networks. J. Commun. Netw-s. Kor. $\mathbf{9}$, 473-481 (2007)

29. X Zhang, Z Zheng, J Liu, X Shen, L-L Xie, in Proceedings of IEEE Global Communications Conference (GLOBECOM). Optimal power allocation and AP deployment in green wireless cooperative communications (IEEE Anaheim, USA, 2012), pp. 4000-4005

30. GB S Farshad, ML, in Proceedings of International Union of Radio Science General Assembly and Scientific Symposium (URSI GASS). A Q-learning game-theory-based algorithm to improve the energy efficiency of a multiple relay-aided network (IEEE Beijing, China, 2014), pp. 1-4

31. L Zhang, Y Liang, Y Xin, Joint beamforming and power allocation for multiple access channels in cognitive radio networks. IEEE J. Sel. Areas Commun. 26, 38-51 (2008)

32. R Madan, N Mehta, A Molisch, J Zhang, Energy-efficient cooperative relaying over fading channels with simple relay selection. IEEE Trans. Wireless Commun. 7, 3013-3025 (2008)

33. G Lim, L Cimini, Energy-efficient cooperative relaying in heterogeneous radio access networks. IEEE Wireless Commun. Lett. 1, 476-479 (2012)

34. Q Guan, F Yu, S Jiang, V Leung, H Mehrvar, Topology control in mobile ad hoc networks with cooperative communications. IEEE Wireless Commun. 19, 74-79 (2012)

35. C Ling, $X$ Yin, SR Boqule, M Garcia-Lozano, in Proceedings of International Union of Radio Science General Assembly and Scientific Symposium (URSI GASS). Optimal power allocation and relay selection in spectrum sharing cooperative networks (IEEE Beijing, China, 2014), pp. 1-4

36. RT Rockafellar, Convex Analysis. (Princeton University Press, USA, 1996)

37. LV Stephen Boyd, Convex Optimization. (Cambridge University Press, UK, 2006)

38. R Kress, Numerical analysis. (World Book Publishing, China, 2003)

39. WC Jakes, Microwave Mobile Communications. (John Wiley \& Sons, USA, 1974)

40. C Komninakis, in Proceedings of IEEE Global Telecommunications Conference (GLOBECOM). A fast and accurate Rayleigh fading simulator (IEEE San Francisco, USA, 2003), pp. 3306-3310

41. H Yu, Y Li, M Kountouris, X Xu, J Wang, in Proceedings of 12th International symposium on Modeling and Optimization in Mobile, Ad Hoc, and Wireless Networks (WiOpt). Energy efficiency analysis of relay-assisted cellular networks using stochastic geometry (IEEE Hammamet, Tunis, 2014), pp. 667-671

42. J Chen, $X$ Chen, $T$ Liu, L Lei, in Proceedings of IEEE international conference on Communications in China (ICCC). Energy-efficient power allocation for secure communications in large-scale MIMO relaying systems (IEEE Shanghai, China, 2014), pp. 385-390

\section{Submit your manuscript to a SpringerOpen ${ }^{\odot}$ journal and benefit from:}

- Convenient online submission

- Rigorous peer review

- Immediate publication on acceptance

- Open access: articles freely available online

- High visibility within the field

- Retaining the copyright to your article 